New Zealand, Institute of Nuclear Science ${ }^{1,2}$

\begin{tabular}{|c|c|c|c|c|}
\hline $\begin{array}{l}\text { Laboratory } \\
\text { number }\end{array}$ & $\begin{array}{l}\text { Published } \\
\text { reference }\end{array}$ & $\begin{array}{cl}\text { Original date or } & \text { Corrected date } \\
\text { other value } & \text { or other value }\end{array}$ & $\partial \mathrm{C}^{14}, \%$ & $\begin{array}{l}\text { A.D./ } \\
\text { B.C. date }\end{array}$ \\
\hline NZ-1 & $111: 119$ & $1820 \pm 150^{\mathrm{s}}$ & & A.D. 130 \\
\hline-2 & $111: 119$ & $3440 \pm 70^{\mathrm{s}}$ & & 1490 B.C. \\
\hline-3 & $111: 119$ & $1640 \pm 70^{\mathrm{s}}$ & & A.D. 310 \\
\hline-4 & $111: 119$ & $1920 \pm 150^{\mathrm{s}}$ & & A.D. 30 \\
\hline-5 & $111: 119$ & $3170 \pm 200$ & & 1220 B.C. \\
\hline-6 & $111: 119$ & $>31,000$ & & \\
\hline-7 & $111: 119$ & $2400 \pm 170$ & & 450 B.C. \\
\hline-8 & $111: 119$ & $2420 \pm 170$ & & 470 B.C. \\
\hline-9 & $111: 120$ & $930 \pm 150$ & & A.D. 1020 \\
\hline-10 & $111: 120$ & $930 \pm 70$ & & A.D. 1020 \\
\hline-11 & $111: 120$ & $9270 \pm 80$ & & 7320 B.C. \\
\hline-12 & $111: 120$ & $20,670 \pm 300$ & & 18,720 B.C. \\
\hline-13 & $111: 120$ & $4720 \pm 70$ & & 2770 B.C. \\
\hline-14 & $111: 120$ & $>37,000$ & & \\
\hline-15 & $111: 121$ & $840 \pm 50$ & & A.D. 1110 \\
\hline-16 & $111: 121$ & $13,870 \pm 250$ & & 11,920 B.C. \\
\hline-17 & $111: 121$ & $>35,000$ & & \\
\hline-18 & $111: 121$ & $>35,000$ & & \\
\hline-19 & $111: 121$ & $>37,000$ & & \\
\hline-20 & $111: 121$ & $>37,000$ & & \\
\hline-21 & $111: 121$ & $>37,000$ & & \\
\hline-22 & $111: 122$ & $>35,000$ & & \\
\hline .23 & $111: 122$ & $>37,000$ & & \\
\hline-24 & $111: 122$ & $3520 \pm 100$ & & 1570 B.C. \\
\hline-25 & $111: 122$ & $>40,000$ & & \\
\hline-26 & $111: 122$ & $2420 \pm 100$ & & 470 B.C. \\
\hline-27 & $111: 122$ & $3720 \pm 100$ & & 1770 B.C. \\
\hline-28 & $111: 122$ & $5920 \pm 100$ & & 3970 B.C. \\
\hline-29 & $111: 122$ & $34,750 \pm 1000$ & & 32,800 B.C. \\
\hline-30 & $111: 123$ & $4470 \pm 100$ & & 2520 B.C. \\
\hline-31 & $111: 123$ & $4400 \pm 100$ & & 2450 B.C. \\
\hline-32 & $111: 123$ & Modern & & \\
\hline .33 & $111: 123$ & $4830 \pm 70$ & & 2880 B.C. \\
\hline-34 & $111: 123$ & $19,200 \pm 500$ & & 17,250 B.C. \\
\hline-37 & $111: 123$ & $1780 \pm 60$ & & A.D. 170 \\
\hline-38 & $111: 123$ & $1800 \pm 70$ & & A.D. 150 \\
\hline-39 & $111: 123$ & $850 \pm 50$ & & A.D. 1100 \\
\hline-40 & $111: 124$ & $>42,000$ & & \\
\hline-41 & $111: 124$ & $20,900 \pm 300$ & & 18,950 B.C. \\
\hline-42 & $111: 124$ & $>40,000$ & & \\
\hline-43 & $111: 124$ & $>42,000$ & & \\
\hline-44 & $111: 124$ & $>42,000$ & & \\
\hline-45 & $111: 124$ & $2370 \pm 70$ & & 420 B.C. \\
\hline-46 & $111: 124$ & $1450 \pm 70$ & & A.D. 500 \\
\hline-47 & $111: 125$ & $660 \pm 60$ & & A.D. 1290 \\
\hline-48 & $111: 125$ & $620 \pm 60$ & & A.D. 1330 \\
\hline .49 & $111: 125$ & $8200 \pm 150$ & & 6250 B.C. \\
\hline-50 & $111: 125$ & $850 \pm 50$ & & A.D. 1100 \\
\hline-51 & $111: 125$ & $230 \pm 60$ & & A.D. 1720 \\
\hline-52 & $111: 125$ & $830 \pm 50$ & & A.D. 1120 \\
\hline-53 & $111: 125$ & $740 \pm 75$ & & A.D. 1210 \\
\hline-54 & $111: 126$ & $390 \pm 60$ & & A.D. 1560 \\
\hline-55 & $111: 126$ & $520 \pm 55$ & & A.D. 1430 \\
\hline-56 & $111: 126$ & $520 \pm 55$ (carbonate) & & A.D. 1430 \\
\hline-56 & $111: 126$ & $550 \pm 55$ (organic) & & A.D. 1400 \\
\hline-57 & $111: 126$ & $600 \pm 60$ & & A.D. 1350 \\
\hline-58 & $111: 126$ & $810 \pm 60$ & & A.D. 1140 \\
\hline-59 & $111: 126$ & $410 \pm 55$ (carbonate) & & A.D. 1540 \\
\hline-59 & $111: 126$ & $400 \pm 55$ (organic) & & A.D. 1550 \\
\hline .60 & $111: 127$ & $450 \pm 60$ & & A.D. 1500 \\
\hline
\end{tabular}


Comprehensive Index, 1950-1965

\begin{tabular}{|c|c|c|c|c|}
\hline $\begin{array}{c}\text { Laboratory } \\
\text { number }\end{array}$ & $\begin{array}{l}\text { Published } \\
\text { reference }\end{array}$ & $\begin{array}{l}\text { Original date or } \\
\quad \text { other value }\end{array}$ & $\begin{array}{l}\text { Corrected date } \\
\text { or other value }\end{array}$ & $\partial \mathrm{C}^{14}, \% 0$ \\
\hline-61 & $111: 127$ & $590 \pm 50$ & & \\
\hline-62 & $111: 127$ & $600 \pm 60$ & & \\
\hline-63 & $111: 127$ & $400 \pm 60$ & & \\
\hline-64 & $111: 127$ & $360 \pm 60$ & & \\
\hline-65 & $111: 127$ & $>45,000$ & & \\
\hline-66 & $111: 127$ & $6570 \pm 100$ & & \\
\hline-67 & $111: 128$ & Modern & & \\
\hline-68 & $111: 128$ & Modern & & \\
\hline-69 & $111: 128$ & $8800 \pm 120$ & & \\
\hline-70 & $111: 128$ & $>48,000$ & & \\
\hline-72 & $111: 128$ & $4620 \pm 80$ & & \\
\hline-73 & $111: 128$ & $4550 \pm 80$ & & \\
\hline-74 & $111: 128$ & $>45,000$ & & \\
\hline-75 & $111: 129$ & $3570 \pm 70$ & & \\
\hline-76 & $111: 129$ & $43,000 \pm 2000$ & & \\
\hline .77 & $111: 129$ & $18,000 \pm 200$ & & \\
\hline-78 & $111: 129$ & $19,800 \pm 300$ & & \\
\hline-79 & $111: 129$ & $>43,000$ & & \\
\hline-80 & $111: 129$ & $>45,000$ & & \\
\hline-81 & $111: 129$ & $9900 \pm 150$ & & \\
\hline-82 & $111: 129$ & $2000 \pm 100$ & & \\
\hline-84 & $111: 129$ & $1550 \pm 80$ & & \\
\hline-85 & $111: 130$ & $>37,000$ & & \\
\hline-86 & $111: 130$ & $940 \pm 70$ & & \\
\hline-87 & $111: 130$ & $>40,000$ & & \\
\hline-88 & $111: 130$ & $15,100 \pm 200$ & & \\
\hline-89 & $111: 130$ & $650 \pm 60$ & & \\
\hline-90 & $111: 130$ & $670 \pm 60$ & & \\
\hline-91 & $111: 130$ & $1020 \pm 60$ & & \\
\hline-92 & $111: 130$ & $>45,000$ & & \\
\hline-93 & $111: 131$ & $6050 \pm 110$ & & \\
\hline-94 & $111: 131$ & $>45,000$ & & \\
\hline-95 & 111:131 & $>42,000$ & & \\
\hline-96 & $111: 131$ & $>40,000$ & & \\
\hline-97 & $111: 131$ & $6050 \pm 80$ & & \\
\hline-98 & $111: 131$ & $10,200 \pm 120$ & & \\
\hline-99 & $111: 131$ & $>45,000$ & & \\
\hline-100 & $111: 131$ & $2200 \pm 60$ & & \\
\hline-101 & $111: 131$ & $2190 \pm 60$ & & \\
\hline-102 & $111: 132$ & $21,500 \pm 350$ & & \\
\hline-103 & $111: 132$ & $21,300 \pm 350$ & & \\
\hline-104 & $111: 132$ & $22,300 \pm 350$ & & \\
\hline-105 & $111: 132$ & $22,800 \pm 350$ & & \\
\hline-106 & $111: 132$ & $37,500 \pm 1600$ & & \\
\hline-107 & $111: 132$ & $1650 \pm 60$ & & \\
\hline-108 & $111: 132$ & $>41,000$ & & \\
\hline-109 & $111: 132$ & $24,000 \pm 500$ & & \\
\hline-110 & $111: 132$ & $27,000 \pm 600$ & & \\
\hline-111 & $111: 132$ & $7230 \pm 100$ & & \\
\hline-112 & $111: 133$ & $11,500 \pm 160$ & & \\
\hline-113 & $111: 133$ & $2030 \pm 100$ & & \\
\hline-114 & $111: 133$ & $1030 \pm 60$ & & \\
\hline-115 & $111: 133$ & $>40,000$ & & \\
\hline-116 & $111: 133$ & $22,300 \pm 350$ & & \\
\hline-117 & $111: 133$ & $6800 \pm 90$ & & \\
\hline-118 & $111: 133$ & $9400 \pm 120$ & & \\
\hline-119 & $111: 134$ & $8000 \pm 150$ & & \\
\hline-120 & $111: 134$ & $6200 \pm 120$ & & \\
\hline-121 & $111: 134$ & $>43.000$ & & \\
\hline
\end{tabular}

A.D./

B.C. date

A.D. 1360

A.D. 1350

A.D. 1550

A.D. 1590

4620 B.C.

6850 B.C.

2670 B.C.

2600 B.C.

1620 B.C.

41,050 B.C.

16,050 B.C.

17,850 B.C.

7950 B.C.

50 B.C.

A.D. 400

A.D. 1010

A.D. 1300

A.D. 1280

A.D. 930

4100 B.C.

4100 B.C.

8250 B.C.

250 B.C.

240 B.C.

19,550 B.C.

19,350 B.C.

20,350 B.C.

20,850 B.C.

35,550 B.C.

A.D. 300

22,050 B.C.

25,050 B.C.

5280 B.C.

9550 B.C.

80 B.C.

A.D. 920

20,350 B.C.

4850 B.C.

7450 B.C.

6050 B.C.

4250 B.C. 


\begin{tabular}{|c|c|c|c|c|}
\hline $\begin{array}{l}\text { Laboratory } \\
\text { number }\end{array}$ & $\begin{array}{c}\text { Published } \\
\text { reference }\end{array}$ & $\begin{array}{l}\text { Original date or } \\
\text { other value }\end{array}$ & $\begin{array}{l}\text { Corrected date } \\
\text { or other value }\end{array}$ & $\partial \mathrm{C}^{14}, \%$ \\
\hline-125 & $111: 134$ & $>45,000$ & & \\
\hline-126 & $\begin{array}{l}111: 134 \\
111: 135\end{array}$ & $\begin{array}{l}>45,000 \\
6620 \pm 100\end{array}$ & & \\
\hline $\begin{array}{l}-127 \\
-128\end{array}$ & $\begin{array}{l}111: 135 \\
111: 135\end{array}$ & $\begin{array}{l}6620 \pm 100 \\
1450 \pm 60\end{array}$ & & \\
\hline $\begin{array}{l}-128 \\
-129\end{array}$ & $111: 135$ & $620 \pm 80$ & & \\
\hline $\begin{array}{l}-129 \\
-130\end{array}$ & $111: 135$ & $<100$ & & \\
\hline-131 & $111: 135$ & $<100$ & & \\
\hline-132 & $111: 135$ & $3320 \pm 120$ & & \\
\hline-133 & $111: 135$ & $320 \pm 60$ & & \\
\hline-134 & $111: 135$ & $765 \pm 30$ & & \\
\hline-135 & $111: 135$ & see NZ-134 & $755 \pm 30$ & \\
\hline-136 & $111: 136$ & $630 \pm 50$ & & \\
\hline-137 & $111: 136$ & $460 \pm 50$ & & \\
\hline-138 & $111: 136$ & $460 \pm 80$ & & \\
\hline-139 & $111: 136$ & $310 \pm 60$ & & \\
\hline-140 & $111: 136$ & $390 \pm 80$ & & \\
\hline-141 & $111: 136$ & $470 \pm 60$ & & \\
\hline-142 & $111: 136$ & $290 \pm 80$ & & \\
\hline-143 & $111: 136$ & $320 \pm 50$ & & \\
\hline-144 & $111: 136$ & $345 \pm 50$ & & \\
\hline-145 & $111: 136$ & $215 \pm 50$ & & \\
\hline-146 & 111:136 & $280 \pm 80$ & & \\
\hline-147 & $111: 137$ & $450 \pm 60$ & & \\
\hline-148 & $111: 137$ & $<160$ & & \\
\hline-149 & $111: 137$ & $310 \pm 60$ & & \\
\hline-150 & $111: 137$ & $19,500 \pm 1000$ & & \\
\hline-151 & $111: 137$ & $27,500 \pm 3000$ & & \\
\hline-152 & $111: 137$ & $<200$ & & \\
\hline-153 & $111: 137$ & $<250$ & & \\
\hline-154 & $111: 137$ & $580 \pm 100$ & & \\
\hline-155 & $111: 137$ & $250 \pm 80$ & & \\
\hline-156 & $111: 137$ & $250 \pm 80$ & & \\
\hline-157 & $111: 138$ & $2270 \pm 100$ & & \\
\hline-158 & $111: 138$ & $1760 \pm 80$ & & \\
\hline-159 & $111: 138$ & $1750 \pm 80$ & & \\
\hline-160 & $111: 138$ & $1300 \pm 80$ & & \\
\hline .161 & $111: 138$ & $1780 \pm 80$ & & \\
\hline-162 & $111: 138$ & $1830 \pm 70$ & & \\
\hline-163 & $111: 138$ & $1840 \pm 50$ & & \\
\hline-164 & $111: 138$ & $1890 \pm 70$ & & \\
\hline-165 & $111: 139$ & $1900 \pm 70$ & & \\
\hline-166 & $111: 139$ & $840 \pm 50$ & & \\
\hline-167 & $111: 139$ & $3110 \pm 70$ & & \\
\hline-168 & $111: 139$ & $1900 \pm 60$ & & \\
\hline-169 & $111: 139$ & $900 \pm 40$ & & \\
\hline-170 & $111: 139$ & $1800 \pm 50$ & & \\
\hline-171 & $111: 139$ & $2650 \pm 150$ & & \\
\hline-172 & $111: 140$ & $1800 \pm 100$ & & \\
\hline-173 & $111: 140$ & $1750 \pm 50$ & & \\
\hline-174 & $111: 140$ & $1800 \pm 100$ & & \\
\hline-175 & $111: 140$ & $1850 \pm 100$ & & \\
\hline-176 & 111:140 & $1900 \pm 70$ & & \\
\hline-177 & $111: 140$ & $2500 \pm 200$ & & \\
\hline-178 & $111: 140$ & $2100 \pm 100$ & & \\
\hline .179 & $111: 140$ & $3420 \pm$ & & \\
\hline
\end{tabular}

A.D./

B.C. date

4670 B.C.

A.D. 500

A.D. 1330

1370 B.C.

A.D. 1630

A.D. 1185

A.D. 1195

A.D. 1320

A.D. 1490

A.D. 1490

A.D. 1640

A.D. 1560

A.D. 1480

A.D. 1660

A.D. 1630

A.D. 1605

A.D. 1735

A.D. 1670

A.D. 1500

A.D. 1640

17,550 B.C. 25,550 B.C.

A.D. 1370

A.D. 1700

A.D. 1700

320 B.C.

A.D. 190

A.D. 200

A.D. 650

A.D. 170

A.D. 120

A.D. 110

A.D. 60

A.D. 50

A.D. 1110

1160 B.C.

A.D. 50

A.D. 1050

A.D. 150

700 B.C.

A.D. 150

A.D. 200

A.D. 150

A.D. 100

A.D. 50

550 B.C.

150 B.C.

1470 B.C. 


\begin{tabular}{|c|c|c|c|c|}
\hline $\begin{array}{l}\text { Laboratory } \\
\text { number }\end{array}$ & $\begin{array}{c}\text { Published } \\
\text { reference }\end{array}$ & $\begin{array}{l}\text { Original date or } \\
\text { other value }\end{array}$ & $\begin{array}{l}\text { Corrected date } \\
\text { or other value }\end{array}$ & $\partial \mathrm{C}^{14}, \%$ \\
\hline-190 & $111: 141$ & $1190 \pm 60$ & & \\
\hline-191 & $111: 142$ & $>45,000$ & & \\
\hline-192 & $111: 142$ & $>40,000$ & & \\
\hline-193 & $111: 142$ & $39,000 \pm 3000$ & & \\
\hline-194 & $111: 142$ & $30,300 \pm 800$ & & \\
\hline-195 & $111: 142$ & $6270 \pm 120$ & & \\
\hline-196 & $111: 142$ & $5400 \pm 80$ & & \\
\hline-197 & $111: 142$ & $>33,000$ & & \\
\hline-198 & $111: 142$ & $29,000 \pm 800$ & & \\
\hline-199 & $111: 143$ & $3740 \pm 100$ & & \\
\hline-200 & $111: 143$ & $390 \pm 60$ & & \\
\hline-201 & $111: 143$ & $<120$ & & \\
\hline-202 & $111: 143$ & $<200$ & & \\
\hline-203 & $111: 143$ & $<200$ & & \\
\hline-204 & $111: 143$ & $<100$ & & \\
\hline-205 & $111: 143$ & $>40,000$ & & \\
\hline .206 & $111: 143$ & $6700 \pm 250$ & & \\
\hline-207 & $111: 143$ & $18,000 \pm 500$ & & \\
\hline-215 & $111: 143$ & $29,000 \pm 1500$ & & \\
\hline-216 & $111: 144$ & $28,000 \pm 1000$ & & \\
\hline-217 & $111: 144$ & $28,000 \pm 1000$ & & \\
\hline-218 & $111: 144$ & $>42,000$ & & \\
\hline-219 & $111: 144$ & $>40,000$ & & \\
\hline .220 & $111: 144$ & $750 \pm 50$ & & \\
\hline-221 & $111: 144$ & $280 \pm 40$ & & \\
\hline-222 & $111: 144$ & $770 \pm 50$ & & \\
\hline-223 & $111: 145$ & $>30,000$ & & \\
\hline-224 & $111: 145$ & $>42,000$ & & \\
\hline-225 & $111: 145$ & $9000 \pm 160$ & & \\
\hline-226 & $111: 145$ & $<200$ & & \\
\hline-227 & $111: 145$ & $>36,000$ & & \\
\hline-265 & $111: 145$ & $980 \pm 60$ & & \\
\hline-266 & $111: 145$ & $1160 \pm 60$ & & \\
\hline-267 & $111: 145$ & $1540 \pm 60$ & & \\
\hline .268 & $111: 146$ & $1760 \pm 70$ & & \\
\hline .269 & $111: 146$ & $2370 \pm 70$ & & \\
\hline-270 & $111: 146$ & $2730 \pm 70$ & & \\
\hline-271 & $111: 146$ & $3150 \pm 80$ & & \\
\hline-272 & $111: 146$ & $3900 \pm 90$ & & \\
\hline-273 & $111: 146$ & $<250$ & & \\
\hline-274 & $111: 146$ & $5520 \pm 70$ & & \\
\hline-275 & $111: 146$ & $7780 \pm 80$ & & \\
\hline-276 & $111: 147$ & $8530 \pm 110$ & & \\
\hline-277 & $111: 147$ & $5270 \pm 80$ & & \\
\hline-278 & $111: 147$ & $1180 \pm 55$ & & \\
\hline-279 & $111: 147$ & $5620 \pm 90$ & & \\
\hline .280 & $111: 147$ & $3720 \pm 85$ & & \\
\hline-281 & $111: 147$ & $9850 \pm 130$ & & \\
\hline-282 & $111: 147$ & $7470 \pm 120$ & & \\
\hline-283 & $111: 148$ & $2920 \pm 100$ & & \\
\hline-284 & $111: 148$ & $3885 \pm 125$ & & \\
\hline-285 & $111: 148$ & $280 \pm 60$ & & \\
\hline-286 & $111: 148$ & $1040 \pm 50$ & & \\
\hline-287 & $111: 148$ & $2800 \pm 80$ & & \\
\hline .288 & $111: 148$ & $2800 \pm 80$ & & \\
\hline-289 & $111: 148$ & $3400 \pm 100$ & & \\
\hline-290 & $111: 148$ & $3950 \pm 100$ & & \\
\hline .291 & $111: 149$ & $2100 \pm 60$ & & \\
\hline-292 & $111: 149$ & $3400 \pm 90$ & & \\
\hline .293 & $111: 149$ & $855 \pm 50$ & & \\
\hline-294 & $111: 149$ & $980 \pm 25$ & & \\
\hline-295 & $111: 149$ & $615 \pm 75$ & & \\
\hline
\end{tabular}

A.D./

B.C. date

A.D. 760

37,050 B.C.

28,350 B.C.

4320 B.C.

3450 B.C.

27,050 B.C.

1790 B.C.

A.D. 1560

4750 B.C.

16,050 B.C.

27,050 B.C.

26,050 B.C.

26,050 B.C.

A.D. 1200

A.D. 1670

A.D. 1180

7050 B.C.

A.D. 970

A.D. 790

A.D. 410

A.D. 190

420 B.C.

780 B.C.

1200 B.C.

1950 B.C.

3570 B.C.

5830 B.C.

6580 B.C.

3320 B.C.

A.D. 770

3670 B.C.

1770 B.C.

7900 B.C.

5520 B.C.

970 B.C.

1935 B.C.

A.D. 1670

A.D. 910

850 B.C.

850 B.C.

1450 B.C.

2000 B.C.

150 B.C.

1450 B.C.

A.D. 1095

A.D. 970

A.D. 1335 


\begin{tabular}{|c|c|c|c|c|}
\hline $\begin{array}{l}\text { Laboratory } \\
\text { number }\end{array}$ & $\begin{array}{l}\text { Published } \\
\text { reference }\end{array}$ & $\begin{array}{l}\text { Original date or } \\
\text { other value }\end{array}$ & $\begin{array}{l}\text { Corrected date } \\
\text { or other value }\end{array}$ & $\partial \mathrm{C}^{14}, \%$ \\
\hline $\begin{array}{l}-296 \\
-297\end{array}$ & $\begin{array}{l}111: 149 \\
111: 149\end{array}$ & $\begin{array}{l}1690 \pm 60 \\
8350 \pm 135\end{array}$ & & \\
\hline $\begin{array}{l}-291 \\
-298\end{array}$ & $111: 150$ & $1570 \pm 65$ & & \\
\hline-299 & $111: 150$ & $2760 \pm 75$ & & \\
\hline-300 & $111: 150$ & $3380 \pm 75$ & & \\
\hline-301 & $111: 150$ & $6560 \pm 100$ & & \\
\hline-302 & $111: 150$ & $9820 \pm 155$ & & \\
\hline-303 & $111: 150$ & $1530 \pm 60$ & & \\
\hline-304 & $111: 150$ & $510 \pm 50$ & & \\
\hline-305 & $111: 150$ & $2040 \pm 60$ & & \\
\hline-306 & $111: 151$ & $3750 \pm 80$ & & \\
\hline-307 & $111: 151$ & $3160 \pm 808$ & & \\
\hline-308 & $111: 151$ & $6980 \pm 100$ & & \\
\hline-309 & $111: 151$ & $4660 \pm 90$ & & \\
\hline-310 & $111: 151$ & $2990 \pm 110$ & & \\
\hline-311 & $111: 151$ & $2460 \pm 70$ & & \\
\hline-312 & $111: 151$ & $735 \pm 55$ & & \\
\hline-313 & $111: 152$ & $6290 \pm 110$ & & \\
\hline-314 & $111: 152$ & $7600 \pm 160$ & & \\
\hline .315 & $111: 152$ & $1510 \pm 55$ & & \\
\hline .316 & $111: 152$ & $2565 \pm 130$ & & \\
\hline-317 & $111: 152$ & $4400 \pm 90$ & & \\
\hline-318 & $111: 152$ & $<120$ & & \\
\hline-319 & $111: 152$ & $3540 \pm 230$ & & \\
\hline-320 & $111: 153$ & $2450 \pm 80$ & & \\
\hline .321 & $111: 153$ & $3250 \pm 80$ & & \\
\hline-322 & $111: 153$ & $4550 \pm 100$ & & \\
\hline-323 & $111: 153$ & $<200$ & & \\
\hline-324 & $111: 153$ & $7090 \pm 115$ & & \\
\hline-325 & $111: 153$ & $4410 \pm 320$ & & \\
\hline-326 & $111: 154$ & $9580 \pm 150$ & & \\
\hline-327 & $111: 154$ & $<200$ & & \\
\hline-328 & $111: 154$ & $930 \pm 165$ & & \\
\hline-329 & $111: 154$ & $16,300 \pm 270$ & & \\
\hline-330 & $111: 154$ & $20,000 \pm 500$ & & \\
\hline-331 & $111: 154$ & $34,000 \pm 1500$ & & \\
\hline .332 & $111: 154$ & $>43,000$ & & \\
\hline .333 & $111: 154$ & $5370 \pm 90$ & & \\
\hline-334 & $111: 155$ & $11,900 \pm 750$ & & \\
\hline-335 & $111: 155$ & $1420 \pm 60$ & & \\
\hline-336 & $111: 155$ & $1750 \pm 60$ & & \\
\hline-337 & $111: 155$ & $1130 \pm 60$ & & \\
\hline-338 & $111: 155$ & $1970 \pm 70$ & & \\
\hline-339 & $111: 155$ & $11,500 \pm 170$ & & \\
\hline-340 & $111: 155$ & $11,900 \pm 200$ & & \\
\hline-341 & $111: 155$ & $31,300 \pm 900$ & & \\
\hline-342 & $111: 156$ & $41,000 \pm 2300$ & & \\
\hline .343 & $111: 156$ & $>39,000$ & & \\
\hline-344 & $111: 156$ & $800 \pm 60$ & & \\
\hline-345 & $111: 156$ & $1680 \pm 60$ & & \\
\hline-346 & $111: 156$ & $1810 \pm 65$ & & \\
\hline $\begin{array}{l}-540 \\
-347\end{array}$ & $111: 156$ & $5610 \pm 90$ & & \\
\hline-348 & $111: 156$ & $>40,000$ & & \\
\hline-349 & $111: 156$ & $37,500 \pm 1900$ & & \\
\hline-350 & $111: 157$ & $>40,000$ & & \\
\hline-351 & $111: 157$ & $>39,800$ & & \\
\hline-352 & $111: 157$ & $1950 \pm 75$ & & \\
\hline-353 & $111: 157$ & $28,000 \pm 600$ & & \\
\hline-354 & $111: 157$ & $640 \pm 50$ & & \\
\hline-355 & $111: 157$ & $600 \pm 50$ & & \\
\hline-356 & $111: 157$ & $<200$ & & \\
\hline-357 & $111: 158$ & $590 \pm 50$ & & \\
\hline
\end{tabular}

A.D./

B.C. date

A.D. 260

6400 B.C.

A.D. 380

810 B.C.

1430 B.C.

4610 B.C.

7870 B.C.

A.D. 420

A.D. 1440

90 B.C.

1800 B.C.

1210 B.C.

5030 B.C.

2710 B.C.

1040 B.C.

510 B.C.

A.D. 1215

4340 B.C.

5650 B.C.

A.D. 440

615 B.C.

2450 B.C.

1590 B.C.

500 B.C.

1300 B.C.

2600 B.C.

5140 B.C.

2460 B.C.

7630 B.C.

A.D. 1020

14,350 B.C.

18,050 B.C.

32,050 B.C.

3420 B.C. 9950 B.C.

A.D. 530

A.D. 200

A.D. 820

20 B.C.

9550 B.C.

9950 B.C.

29,350 B.C.

39,050 B.C.

A.D. 1150

A.D. 270

A.D. 140

3660 B.C.

35,550 B.C.

A.D. 1

26,050 B.C.

A.D. 1310

A.D. 1350

A.D. 1360 


\begin{tabular}{|c|c|c|c|c|}
\hline $\begin{array}{l}\text { Laboratory } \\
\text { number }\end{array}$ & $\begin{array}{l}\text { Published } \\
\text { reference }\end{array}$ & $\begin{array}{l}\text { Original date or } \\
\quad \text { other value }\end{array}$ & $\begin{array}{l}\text { Corrected date } \\
\text { or other value }\end{array}$ & $\begin{array}{l}\text { A.D./ } \\
\text { B.C. date }\end{array}$ \\
\hline-358 & $111: 158$ & $810 \pm 50$ & & A.D. 1140 \\
\hline-359 & $111: 158$ & $650 \pm 50$ & & A.D. 1300 \\
\hline-360 & $111: 158$ & $240 \pm 50$ & & A.D. 1710 \\
\hline-361 & $111: 158$ & $1880 \pm 60$ & & A.D. 70 \\
\hline-362 & $111: 158$ & $1850 \pm 50$ & & A.D. 100 \\
\hline-363 & $111: 158$ & $1950 \pm 120$ & & A.D. 1 \\
\hline-364 & $111: 158$ & $4800 \pm 100$ & & 2850 B.C. \\
\hline-365 & $111: 158$ & $3240 \pm 80$ & & 1290 B.C. \\
\hline-366 & $111: 159$ & $15,000 \pm 1500$ & & 13,050 B.C. \\
\hline-367 & $111: 159$ & $370 \pm 45$ & & A.D. 1580 \\
\hline-368 & $111: 159$ & $5090 \pm 200$ & & 3140 B.C. \\
\hline-369 & $111: 159$ & $5020 \pm 120$ & & 3070 B.C. \\
\hline-370 & $111: 159$ & $8390 \pm 135$ & & 6440 B.C. \\
\hline-371 & $111: 159$ & $385 \pm 50$ & & A.D. 1565 \\
\hline-372 & $111: 160$ & $8020 \pm 130$ & & 6070 B.C. \\
\hline-373 & $111: 160$ & $39,600 \pm 2000$ & & 37,650 B.C. \\
\hline-374 & $111: 160$ & $760 \pm 50$ & & A.D. 1190 \\
\hline-375 & $111: 160$ & $715 \pm 50$ & & A.D. 1235 \\
\hline-376 & $111: 160$ & $1850 \pm 80$ & & A.D. 100 \\
\hline-377 & $111: 160$ & $1915 \pm 65$ & & A.D. 35 \\
\hline-378 & $111: 160$ & $>40,000$ & & \\
\hline-379 & $111: 161$ & $>34,000$ & & \\
\hline-380 & $111: 161$ & $7400 \pm 200$ & & 5450 B.C. \\
\hline-381 & $111: 161$ & $11,100 \pm 130$ & & 9150 B.C. \\
\hline
\end{tabular}

${ }^{1}$ Modern standard, both for terrestrial organic matter and for shallow-water marine carbonates, is age-corrected pre-industrial wood, not significantly different from $95 \%$ of NBS oxalic-acid standard. Shallow-water shells are believed not to require a special correction for isotopic fractionation.

${ }^{2}$ Dates as published in ref. 111 are believed not to require correction. References to original New Zealand date lists I-V are given in ref. 111.

\section{Humble, Esso Production Research Company ${ }^{1}$}

\begin{tabular}{|c|c|c|c|c|c|}
\hline $\begin{array}{c}\text { Laboratory } \\
\text { number }\end{array}$ & $\begin{array}{l}\text { Published } \\
\text { reference }\end{array}$ & $\begin{array}{l}\text { Original date or } \\
\quad \text { other value }\end{array}$ & $\begin{array}{l}\text { Corrected date } \\
\text { or other value }\end{array}$ & $\partial \mathrm{C}^{14}, \% 0$ & $\begin{array}{c}\text { A.D./ } \\
\text { B.C. date }\end{array}$ \\
\hline $0-1 A^{2}$ & $50: 922$ & $>35,000$ & & & \\
\hline $\begin{array}{l}-4 A^{2} \\
-5 A^{2}\end{array}$ & $\begin{array}{l}50: 922 \\
50: 921\end{array}$ & $\begin{array}{c}>35,000 \\
17500 \pm 2000\end{array}$ & & & \\
\hline$-6^{2}$ & $\begin{array}{l}50: 921 \\
50: 920\end{array}$ & $\begin{array}{c}1,500 \pm 2000 \\
2520 \pm 110\end{array}$ & & & $\begin{array}{l}15,550 \text { B.C. } \\
570 \text { B.C. }\end{array}$ \\
\hline$-7^{2}$ & $50: 921$ & $2750 \pm 110$ & & & 800 B.C. \\
\hline$-7 A^{2}$ & $50: 920$ & $1900 \pm 150$ & & & A.D. 50 \\
\hline$-8^{2}$ & $50: 921$ & $1220 \pm 100$ & & & A.D. 730 \\
\hline$-9^{2}$ & $50: 921$ & $520 \pm 100$ & & & 1430 A.D. \\
\hline$-11 A^{2}$ & $50: 921$ & $6520 \pm 300$ & & & 4570 B.C. \\
\hline-12 & $49: 147$ & $1130 \pm 100$ & see $0-27$ & & \\
\hline$-12 A^{2}$ & $50: 921$ & $2750 \pm 200$ & see $0-22$ & & 800 B.C. \\
\hline$-13 A^{2}$ & $50: 921$ & $3200 \pm 120$ & see $0-29$ & & \\
\hline$-14 A^{2}$ & $50: 921$ & $4300 \pm 200$ & & & 2350 B.C. \\
\hline$-22^{2}$ & $50: 921$ & $2800 \pm 110$ & $2775 \pm 110 \mathrm{c}$ & & 825 B.C. \\
\hline $\begin{array}{l}-23 \\
-24\end{array}$ & $\begin{array}{l}49: 147 \\
49: 147\end{array}$ & $\begin{array}{r}980 \pm 100 \\
1410 \pm 100\end{array}$ & ee 0.27 & & A.D 540 \\
\hline-25 & $49: 147$ & $1420 \pm 100$ & & & A.D. 530 \\
\hline-26 & $49: 148$ & $1180 \pm 100$ & & & A.D. 770 \\
\hline-27 & $49: 147$ & $1080 \pm 100$ & $1050 \pm 100 \mathrm{c}$ & & A.D. 900 \\
\hline$-28^{2}$ & $49: 148$ & $1900 \pm 110$ & & & A.D. 50 \\
\hline$-29^{2}$ & $50: 921$ & $2900 \pm 200$ & $3150 \pm 120 \mathrm{c}$ & & 1200 B.C. \\
\hline-30 & $49: 148$ & $2200 \pm 110$ & & & 250 B.C. \\
\hline$-39^{2}$ & $49: 148$ & $200 \pm 100$ & see 0.44 & & \\
\hline$-41^{2}$ & $49: 149$ & $2560 \pm 110$ & & & 610 B.C. \\
\hline
\end{tabular}

